\title{
La participation au centre du montage Quelques pistes pour l'équité et la résilience des forums multipartites
}

\author{
Anne M. Larson et Juan Pablo Sarmiento Barletti
}

\section{Points essentiels}

- Face à l'urgence de la situation climatique et de la nécessité de transformer les trajectoires de développement, les forums multipartites (multi-stakeholder forums ou MSF en anglais) apparaissent de plus en plus essentiels pour que s'instaure une collaboration entre différents niveaux de gouvernement et diverses circonscriptions.

- Une revue de la littérature spécialisée révèle qu'un changement d'orientation s'impose pour que ces forums soient plus équitables et résilients. En effet, au lieu de mettre l'accent sur les modalités de montage des projets, il vaudrait mieux réfléchir à la participation en vue de répondre à une situation ou à un contexte spécifiques.

- Pour mettre la participation au centre du montage, il convient d'associer des approches descendantes et ascendantes, en commençant par une période consacrée à la recherche scientifique et aux réunions aux échelons supérieurs pour comprendre les difficultés potentielles qui peuvent se poser au niveau local aux pilotes de la mise en œuvre d'un projet dans le contexte général d'un site.

- II s'agit d'un processus adaptable qui repose sur la participation et l'engagement, qui favorise un esprit de coapprentissage entre tous les acteurs, et suscite le respect mutuel et la confiance au fil du temps.

- Cette approche a plus de chance de produire des résultats équitables et d'aboutir à la résilience face au changement ou aux problèmes. Elle n'est ni assujettie la nature de plus en plus court-termiste des financements attribués par les bailleurs de fonds, ni uniquement alignée sur des indicateurs d'impact simples et quantitatifs.

\section{Introduction}

Cet Infobrief présente une partie des résultats dégagés d'une revue de la littérature spécialisée consacrée aux forums multipartites ${ }^{1}$ (multistakeholder forums ou MSF en anglais) infranationaux qui ont été mis en place pour s'atteler aux défis de l'utilisation des terres et des changements y afférents. Cette étude, appelée Realist Synthesis Review (RSR), c'est-à-dire synthèse réaliste ${ }^{2}$, s'est appuyée sur des méthodes systématiques pour sélectionner et analyser les cas, afin d'examiner comment une situation ou un contexte spécifiques (p. ex. l'organisation et les institutions sociales et politiques, les questions de genre, I'histoire, les ressources) affecte la performance de ces forums ${ }^{3}$. Cette analyse a mis en lumière un problème tout aussi important : la question de la participation des parties prenantes de manière à agir sur ce contexte et, par conséquent, à produire de meilleurs résultats.

\footnotetext{
À quoi prennent part ces « parties prenantes » ? La réponse à cette question détermine qui est invité à la table de réunion, et cela peut influer sur les solutions possibles. Pour les besoins de cette étude, nous essayons d'employer ce terme et celui d'acteurs de manière interchangeable, et soulignons que la définition des personnes à inviter autour de la table est une variable centrale de l'analyse.

2 Voir Nilsson et al. 2016 et McClain et al. 2018 qui donnent d'autres exemples. 3 Voir Sarmiento Barletti et al. 2020 (revue), 2018 (protocole) et le document d'accompagnement (sur le contexte) Sarmiento Barletti et Larson 2020.
}

Nous définissons ces forums multipartites comme des espaces interactifs organisés spécialement pour rassembler un éventail de parties prenantes afin qu'elles puissent participer au dialogue, à la prise de décisions et/ou à la mise en œuvre en vue de remédier à un problème relatif à la terre ou aux ressources ou d'atteindre un objectif commun. Ces plateformes participatives ont fait l'objet d'un regain d'attention de la part des instances de décision et des praticiens du développement et de la conservation étant donné l'urgence de la situation climatique et de la nécessité de transformer les trajectoires de développement. Ce résumé vise à guider ceux qui conçoivent et mettent en œuvre des forums multipartites, ainsi que les bailleurs de fonds et autres organisations qui les financent. Comme l'indique cette étude, il ne faut pas oublier que les démarches participatives de la conservation et du développement n'ont rien de nouveau et qu'il y a beaucoup à apprendre des expériences du passé.

Bien que l'axe principal de cette revue de littérature soit les effets du contexte sur les résultats obtenus par ces forums, les grands enseignements dégagés de cette analyse portent sur l'approche de ce contexte. Cette revue de littérature a révélé que les forums les plus aptes à réaliser les résultats visés étaient ceux qui étaient reconnus dans le cadre d'un processus plus vaste ayant pour objet la transformation des pratiques à divers niveaux. Ces forums prévoyaient une période consacrée à la recherche scientifique et aux réunions aux échelons 
supérieurs ( $p$. ex. les décideurs des pouvoirs publics ou des ONG qui peuvent faciliter le changement ou bien y faire obstacle) pour repérer les blocages éventuels et les capacités de coopération qui existent avec les pilotes de la mise en œuvre sur le plan local. Ces forums ont permis de susciter le consensus et l'engagement depuis les échelons supérieurs (ainsi que la volonté politique) et ont été conçus comme des espaces d'apprentissage adaptatif 4

Si la compréhension du contexte est essentielle pour produire des initiatives plus résilientes, les cas étudiés font apparaître la nécessité d'insister sur la participation au centre du montage plutôt que sur les modalités de montage des projets. Cela signifie qu'au lieu d'envisager des travaux de manière passive dans le contexte ou autour de celuici, il est préférable de faire participer les parties prenantes de façon à agir activement sur chaque contexte distinct, quelles que soient ses caractéristiques, lors du montage des projets comme lors de leur mise en œuvre. Cet Infobrief expose les recherches scientifiques et les enseignements dégagés qui ont conduit à formuler cette proposition de mettre la participation au centre du montage.

\section{Les forums multipartites : le pluralisme en action ou juste une question de cases à cocher?}

Cette revue de littérature concerne les forums créés au niveau infranational et qui comportent au moins un participant des pouvoirs publics et un représentant d'un autre secteur. Les forums infranationaux ont été choisis pour trois raisons. Premièrement, l'analyse actuelle des forums multipartites d'une part, et de l'utilisation des terres et des changements y afférents d'autre part, se focalise sur les initiatives internationales (p. ex. la Table ronde pour le soja responsable et la Table ronde pour une huile de palme durable). Deuxièmement, les forums infranationaux sont plus proches des territoires et des parties prenantes affectés par la planification, la gestion et le changement d'utilisation des terres dans lesquels ils sont impliqués. Troisièmement, cette analyse répond à l'intérêt croissant du monde universitaire et scientifique, comme des praticiens pour les approches juridictionnelles dans la lutte contre le changement climatique et la déforestation (Boyd et al. 2018; Stickler et al. 2018).

Le nombre en hausse de ces forums témoigne d'une prise de conscience que les problèmes environnementaux ne pourront être réglés sans la participation effective des acteurs qui déterminent sur le terrain les pratiques d'utilisation des terres, et aussi que ces problèmes ne se résoudront pas au sein d'une communauté de conservation lorsque les facteurs à l'œuvre se trouvent dans d'autres secteurs. Les approches les plus répandues - revenant souvent à maintenir le statu quo - sont en général descendantes, ciblant un seul secteur sous la houlette d'experts. Par ailleurs, les études scientifiques réalisées par le Centre de recherche forestière internationale (CIFOR) sur la

4 Dans l'apprentissage adaptatif, les agents en jeu s'adaptent eux-mêmes, à partir de l'acceptation du fait que nul ne sait tout et que leurs idées peuvent être fausses. Cela conduit par conséquent à réfléchir et à revoir les stratégies, les activités et les principes de départ qui les sous-tendent. gouvernance à plusieurs niveaux $x^{5}$ ont permis de découvrir que les ONG et les bailleurs de fonds en particulier tendaient à considérer le forum multipartite comme un élément clé de la solution à certains problèmes comme la dégradation des terres (Ravikumar et al. 2018). Des études universitaires récentes vont dans le sens de la mise en œuvre de ces approches (Kusters et al. 2018). Ce regain d'intérêt pour les plateformes participatives demande une évaluation des enseignements dégagés de la recherche scientifique et de l'expérience de leur mise en œuvre sur plusieurs dizaines d'années (p. ex. Chambers 1983 ; Cornwall 2004).

Les analystes conviennent du défi que posent pour la conservation et le développement les inégalités de pouvoir entre les diverses parties prenantes, mais leur avis diverge sur la capacité des processus participatifs, tels que les forums multipartites, à les transformer. Certains chercheurs voient le potentiel de ces plateformes à promouvoir la prise de décisions transversale (transsectorielle et relative à plusieurs sujets) et une plus grande équité pour les populations locales. De ce point de vue, en réunissant différentes parties prenantes, ces processus participatifs peuvent remédier aux inégalités de pouvoir entre les participants. Ils peuvent aussi déboucher sur des solutions plus acceptables pour les acteurs locaux que celles découlant de décisions prises en haut lieu ou lors de négociations bilatérales. Par ailleurs, ces processus permettent aux instances de décision et aux autres participants de comprendre les points de vue de ceux qui sont les plus touchés par les décisions et les orientations relatives à l'utilisation des terres, tout en impliquant les agents ayant une incidence sur la mise en œuvre et, par conséquent, sur les résultats (Faysse et al. 2006). Cet optimisme, qui n'est pas toujours étayé par des éléments objectifs, s'observe aussi dans les orientations et les pratiques, car bon nombre de bailleurs de fonds et de praticiens insistent sur l'importance de la participation des parties prenantes dans les processus décisionnels concernant l'utilisation des terres et les changements y afférents. Les populations locales, surtout les organisations autochtones, réclament aussi cette participation qui est, en fait, une obligation en vertu du droit internationalement reconnu au « consentement préalable, donné librement et en connaissance de cause » (Espinoza Llanos et Feather 2011).

D'autres analystes avancent que la participation habituelle occulte des montages de gouvernance qui ne règlent pas les problèmes d'inégalité entre les participants, voire même les renforcent (Cornwall 2004). Il est essentiel de ce côté du débat de véritablement prendre conscience des déséquilibres de pouvoir qui existent entre les participants, en termes d'accès non seulement aux ressources économiques et naturelles, mais aussi aux connaissances techniques et quant à la possibilité d'être invité à participer dès le départ (Young 2000). Selon ces érudits, les accords signés ou les résultats obtenus reposent en général sur la conformité volontaire, n'ont pas de force exécutoire et ne sont pas assortis de sanctions, ce qui peut créer des conflits entre les parties prenantes qui participent ou aggraver ceux qui existeraient déjà (Mena et Palazzo 2012). D'autres analystes affirment que ces plateformes sont rarement examinées de près, ou bien épisodiquement, et exigent des méthodes de suivi plus nuancées et élaborées en mode participatif (Kusters et al. 2018).

5 Voir https://www.cifor.org/gcs/modules/multilevel-governance/ 
Malgré cette analyse, ces plateformes collaboratives sont toujours considérées comme ayant un grand potentiel transformationnel. Et bien qu'une transition vers un modèle multipartite sérieux soit louable, de nombreuses initiatives participatives se sont avérées, au moins en partie, des « exercices de cases à cocher » pour respecter les obligations juridiques et satisfaire les demandes des bailleurs de fonds, ou pour légitimer des décisions déjà prises ou ayant perdu leur d'intérêt. Partant du constat que « le contexte a de l'importance » dans les initiatives de conservation et de développement, l'équipe qui s'est consacrée à cette revue de littérature a cherché à en comprendre les effets sur les forums afin d'en tirer des leçons de résilience.

\section{Méthode}

Cette revue de littérature s'est déroulée en cinq phases qui sont expliquées en détail dans le protocole (Sarmiento Barletti et al. 2018).
La première phase a consisté en une recherche initiale systématique, et la deuxième a permis de faire le tri parmi les 984 articles réunis pour n'en garder que 124, c'est-à-dire ceux qui remplissaient les critères de forums multipartites infranationaux comportant au moins un acteur des pouvoirs publics et un représentant d'un autre secteur. La troisième phase était la synthèse des «théories de programme » extraites des articles retenus (voir ci-dessous) en considérant comment chaque forum « aurait dû » fonctionner et selon quels dispositifs. Au cours de la quatrième phase, la recherche a dépassé le cadre de ces articles pour comprendre les principaux facteurs contextuels qui conditionnaient chaque cas (42 articles). La cinquième et dernière phase a donné lieu à la synthèse des 16 articles restants et des 19 études de cas au total (présentant des informations suffisamment approfondies) pour dégager un type de contexte, de dispositif et de résultats en fonction de la théorie de la participation à la base de chaque programme (Tableau 1).

\section{Tableau 1. Les études de cas en bref}

\begin{tabular}{|c|c|c|c|c|c|}
\hline Cas & Titre court & $\begin{array}{l}\text { Référence } \\
\text { bibliographique }\end{array}$ & Modèle(s) & $\begin{array}{l}\text { Porteur de } \\
\text { projet }\end{array}$ & Participants \\
\hline $\begin{array}{l}1 \text { Gestion forestière } \\
\text { conjointe à Gadabanikilo } \\
\text { (Inde) }\end{array}$ & $\begin{array}{l}\text { 1/Gadabanikilo } \\
\text { JFM }\end{array}$ & Nayak et Berkes 2008 & $\begin{array}{l}\text { Durabilité et moyens } \\
\text { de subsistance } \\
\text { (développement) }\end{array}$ & Pouvoirs publics & $\begin{array}{l}\text { Communauté, } \\
\text { pouvoirs publics }\end{array}$ \\
\hline $\begin{array}{l}2 \text { Gestion forestière } \\
\text { conjointe à Uttaranchal } \\
\text { (Inde) }\end{array}$ & $\begin{array}{l}\text { 2/Uttaranchal } \\
\text { JFM }\end{array}$ & Mohanty 2004 & $\begin{array}{l}\text { Durabilité et moyens } \\
\text { de subsistance } \\
\text { (développement) }\end{array}$ & Pouvoirs publics & $\begin{array}{l}\text { Communauté, } \\
\text { pouvoirs publics }\end{array}$ \\
\hline $\begin{array}{l}3 \text { Gestion conjointe de } \\
\text { l'aménagement forestier à } \\
\text { Karnataka (Inde) }\end{array}$ & $\begin{array}{l}\text { 3/Karnataka } \\
\text { JFPM }\end{array}$ & Martin et Lemon 2001 & $\begin{array}{l}\text { Durabilité et moyens } \\
\text { de subsistance } \\
\text { (développement) }\end{array}$ & $\begin{array}{l}\text { Pouvoirs publics et } \\
\text { bailleur de fonds }\end{array}$ & $\begin{array}{l}\text { Communauté, } \\
\text { pouvoirs publics, ONG }\end{array}$ \\
\hline $\begin{array}{l}3 \text { Gestion conjointe de } \\
\text { l'aménagement forestier à } \\
\text { Karnataka (Inde) }\end{array}$ & $\begin{array}{l}\text { 3/Karnataka } \\
\text { JFPM }\end{array}$ & Martin et Lemon 2001 & $\begin{array}{l}\text { Durabilité et moyens } \\
\text { de subsistance } \\
\text { (développement) }\end{array}$ & $\begin{array}{l}\text { Pouvoirs publics et } \\
\text { bailleur de fonds }\end{array}$ & $\begin{array}{l}\text { Communauté, } \\
\text { pouvoirs publics, ONG }\end{array}$ \\
\hline $\begin{array}{l}4 \text { Gestion forestière } \\
\text { conjointe à Karnataka (Inde) }\end{array}$ & $\begin{array}{l}\text { 4/Karnataka } \\
\text { JFM }\end{array}$ & Martin et Lemon 2001 & $\begin{array}{l}\text { Durabilité et moyens } \\
\text { de subsistance } \\
\text { (développement) }\end{array}$ & Pouvoirs publics & $\begin{array}{l}\text { Communauté, } \\
\text { pouvoirs publics, ONG }\end{array}$ \\
\hline $\begin{array}{l}5 \text { Programme forestier } \\
\text { communautaire (Népal) }\end{array}$ & 5/Népal CFP & McDougall et al. 2013 & $\begin{array}{l}\text { Durabilité et } \\
\text { Participation }\end{array}$ & Pouvoirs publics & $\begin{array}{l}\text { Communauté, } \\
\text { pouvoirs publics, ONG }\end{array}$ \\
\hline $\begin{array}{l}6 \text { Bangkok Espaces verts } \\
\text { urbains (Thaïlande) }\end{array}$ & $\begin{array}{l}\text { 6/Bangkok } \\
\text { Green }\end{array}$ & Stringer et al. 2006 & $\begin{array}{l}\text { Durabilité et } \\
\text { Participation }\end{array}$ & ONG & $\begin{array}{l}\text { Communauté, } \\
\text { pouvoirs publics, ONG }\end{array}$ \\
\hline $\begin{array}{l}7 \text { Forêt modèle de Campo- } \\
\text { Ma'an (Cameroun) }\end{array}$ & $\begin{array}{l}\text { 7/Campo- } \\
\text { Ma'an MF }\end{array}$ & Jum et al. 2007 & $\begin{array}{l}\text { Durabilité et } \\
\text { Participation }\end{array}$ & ONG & $\begin{array}{l}\text { Communauté, } \\
\text { pouvoirs publics, ONG }\end{array}$ \\
\hline $\begin{array}{l}8 \text { Forêt modèle de Dja et } \\
\text { Mpomo (Cameroun) }\end{array}$ & $\begin{array}{l}\text { 8/Dja et } \\
\text { Mpomo MF }\end{array}$ & Jum et al. 2007 & $\begin{array}{l}\text { Durabilité et } \\
\text { Participation }\end{array}$ & ONG & $\begin{array}{l}\text { Communauté, } \\
\text { pouvoirs publics, ONG }\end{array}$ \\
\hline $\begin{array}{l}9 \text { Projet de la réserve de } \\
\text { développement durable de } \\
\text { Juma (Brésil) }\end{array}$ & 9/Juma REDD+ & Gebara 2013 & $\begin{array}{l}\text { Moyens de subsistance } \\
\text { (développement) } \\
\text { et coordination à } \\
\text { plusieurs niveaux }\end{array}$ & $\begin{array}{l}\text { ONG et secteur } \\
\text { privé }\end{array}$ & $\begin{array}{l}\text { Communauté, } \\
\text { pouvoirs publics, } \\
\text { ONG, secteur privé }\end{array}$ \\
\hline $\begin{array}{l}10 \text { Projet REDD+ d'Oddar } \\
\text { Meanchey (Cambodge) }\end{array}$ & $\begin{array}{l}\text { 10/Oddar } \\
\text { Meanchey } \\
\text { REDD+ }\end{array}$ & Pasgaard 2015 & $\begin{array}{l}\text { Moyens de subsistance } \\
\text { (développement) } \\
\text { et coordination à } \\
\text { plusieurs niveaux }\end{array}$ & $\begin{array}{l}\text { ONG et bailleur de } \\
\text { fonds }\end{array}$ & $\begin{array}{l}\text { Communauté, } \\
\text { pouvoirs publics, } \\
\text { ONG, secteur privé }\end{array}$ \\
\hline $\begin{array}{l}11 \text { Forêt nationale de Finger } \\
\text { Lakes (États-Unis) }\end{array}$ & $\begin{array}{l}11 / \text { Finger } \\
\text { Lakes }\end{array}$ & Twarkins et al. 2001 & Participation & Pouvoirs publics & $\begin{array}{l}\text { Communauté, } \\
\text { pouvoirs publics }\end{array}$ \\
\hline
\end{tabular}


Tableau 1. Les études de cas en bref (cont.)

\begin{tabular}{|c|c|c|c|c|c|}
\hline Cas & Titre court & $\begin{array}{l}\text { Référence } \\
\text { bibliographique }\end{array}$ & Modèle(s) & $\begin{array}{l}\text { Porteur de } \\
\text { projet }\end{array}$ & Participants \\
\hline $\begin{array}{l}12 \text { Comités de coordination } \\
\text { des forêts de district (Népal) }\end{array}$ & 12/Népal DFCC & Rana et al. 2009 & Participation & Pouvoirs publics & $\begin{array}{l}\text { Communauté, } \\
\text { pouvoirs publics }\end{array}$ \\
\hline $\begin{array}{l}13 \text { Aire protégée de Hin } \\
\text { Nam No (Laos) }\end{array}$ & $\begin{array}{l}\text { 13/Hin Nam } \\
\text { No }\end{array}$ & de Koning et al. 2017 & $\begin{array}{l}\text { Participation et } \\
\text { coordination à } \\
\text { plusieurs niveaux }\end{array}$ & $\begin{array}{l}\text { Pouvoirs publics et } \\
\text { bailleur de fonds }\end{array}$ & $\begin{array}{l}\text { Communauté, } \\
\text { pouvoirs publics }\end{array}$ \\
\hline $\begin{array}{l}14 \text { Forêt modèle de } \\
\text { Vilhelmina (Suède) }\end{array}$ & $\begin{array}{l}\text { 14/Vilhelmina } \\
\text { MF }\end{array}$ & Klenk et al. 2013 & $\begin{array}{l}\text { Participation et } \\
\text { coordination à } \\
\text { plusieurs niveaux }\end{array}$ & ONG & $\begin{array}{l}\text { Communauté, } \\
\text { pouvoirs publics, } \\
\text { ONG, secteur privé }\end{array}$ \\
\hline $\begin{array}{l}15 \text { Nusa Tenggara Barat } \\
\text { (Indonésie) }\end{array}$ & $\begin{array}{l}\text { 15/Nusa } \\
\text { Tenggara Barat }\end{array}$ & Butler et al. 2016 & $\begin{array}{l}\text { Participation et } \\
\text { coordination à } \\
\text { plusieurs niveaux }\end{array}$ & ONG & $\begin{array}{l}\text { Communauté, } \\
\text { pouvoirs publics, ONG }\end{array}$ \\
\hline $\begin{array}{l}16 \text { Parc d'État de lîle de } \\
\text { Cardoso (Brésil) }\end{array}$ & $\begin{array}{l}\text { 16/île de } \\
\text { Cardoso }\end{array}$ & $\begin{array}{l}\text { Sessin-Dilascio et al., } \\
2015\end{array}$ & $\begin{array}{l}\text { Moyens de subsistance } \\
\text { (développement) et } \\
\text { participation }\end{array}$ & Pouvoirs publics & $\begin{array}{l}\text { Communauté, } \\
\text { pouvoirs publics }\end{array}$ \\
\hline $\begin{array}{l}17 \text { Forêt modèle du Prince } \\
\text { Albert (Canada) }\end{array}$ & $\begin{array}{l}\text { 17/Prince } \\
\text { Albert MF }\end{array}$ & Klenk et al. 2013 & $\begin{array}{l}\text { Participation et } \\
\text { coordination à } \\
\text { plusieurs niveaux }\end{array}$ & ONG & $\begin{array}{l}\text { Communauté, } \\
\text { pouvoirs publics, } \\
\text { ONG, secteur privé }\end{array}$ \\
\hline $\begin{array}{l}18 \text { Forum régional sur } \\
\text { le papillon monarque } \\
\text { (Mexique) }\end{array}$ & $\begin{array}{l}\text { 18/Papillon } \\
\text { monarque }\end{array}$ & Brenner et Job 2012 & $\begin{array}{l}\text { Moyens de subsistance } \\
\text { (développement) } \\
\text { et coordination à } \\
\text { plusieurs niveaux }\end{array}$ & Pouvoirs publics & $\begin{array}{l}\text { Communauté, } \\
\text { pouvoirs publics, } \\
\text { ONG, secteur privé }\end{array}$ \\
\hline $\begin{array}{l}19 \text { Forêt modèle du } \\
\text { Manitoba (Canada) }\end{array}$ & $\begin{array}{l}\text { 19/Manitoba } \\
\text { MF }\end{array}$ & Parkins et al. 2016 & $\begin{array}{l}\text { Participation et } \\
\text { coordination à } \\
\text { plusieurs niveaux }\end{array}$ & ONG & $\begin{array}{l}\text { Communauté, } \\
\text { pouvoirs publics, ONG }\end{array}$ \\
\hline
\end{tabular}

\section{Pourquoi organiser des forums multipartites?}

Les « théories de programme » représentent différents modèles, pouvant se recouper, basés sur des priorités et des principes divers, pour encourager une utilisation durable des terres grâce à la participation et à l'engagement (Tableau 2). Dans chaque démarche, le rôle assigné au forum multipartite varie, mais le but est identique, c'est-à-dire obtenir « l'adhésion » de la population locale avec pour fil conducteur les axes suivants : durabilité, moyens de subsistance, participation et coordination à plusieurs niveaux.

\section{Durabilité}

Les forums ayant opté pour cette approche se proposent d'accroître la durabilité et l'inclusion sociale en faisant participer la population locale aux processus décisionnels ou aux organes de gestion visant une utilisation plus durable des terres. Dans ces initiatives, la durabilité est vue comme un concept positif auquel les communautés locales vont adhérer quand elles l'auront compris grâce à leur participation, ce qui est quelque peu limité par ailleurs. Huit cas reposaient sur cette approche ${ }^{6}$.

\section{Moyens de subsistance (c.-à-d. développement)}

En vertu de ce modèle, les organisateurs du forum multipartite pensent que la conservation, ou des pratiques plus durables, feront probablement perdre des moyens de subsistance qui devront être compensés par de nouvelles possibilités d'activités lucratives. Les huit cas suivant cette approche comportaient un dispositif destiné à générer de nouveaux revenus ou bénéfices à partir d'une utilisation plus durable des terres, qui pallieront les pertes subies par la population locale à cause du changement de pratiques ${ }^{7}$. Par ailleurs, selon ce modèle, la participation aux prises de décision qui les concernent devrait motiver les parties prenantes à suivre la voie proposée.

\section{Participation}

Les partisans de cette approche pour les forums multipartites considèrent qu'une utilisation plus durable des terres est possible si I'on accorde aux communautés plus de contrôle sur les ressources naturelles grâce à la prise en compte des institutions locales et à leur officialisation. II est probable que cette approche prévoie un certain type de cogestion ou de coapprentissage. Elle est censée déboucher sur une utilisation plus durable des terres, bénéfique

6 1/Gadabanikilo JFM, 2/Uttaranchal JFM, 3/Karnataka JFPM, 4/Karnataka JFM, 5/Népal CFP, 6/Bangkok Green, 7/Campo-Ma'an MF,

8/Dja et Mpomo MF.

7 1/Gadabanikilo JFM, 2/Uttaranchal JFM, 3/Karnataka JFPM, 4/Karnataka JFM, 9/Juma REDD+, 10/Oddar Meanchey REDD+, 16/île de Cardoso, 18/

Papillon monarque. 


\section{Tableau 2. Modèles de participation}

\begin{tabular}{lll}
\hline Modèle & Dispositif & Résultat visé \\
\hline $\begin{array}{l}\text { Durabilité - cherche à conjuguer les objectifs relatifs au } \\
\text { changement durable d'utilisation des terres, aux moyens } \\
\text { de subsistance et à l'inclusion sociale. }\end{array}$ & $\begin{array}{l}\text { Inclure la population locale dans les } \\
\text { initiatives de durabilité pour la motiver } \\
\text { à adopter les pratiques proposées. }\end{array}$ & $\begin{array}{l}\text { Utilisation plus durable des terres, réduction de } \\
\text { la vulnérabilité de la population locale, plus de } \\
\text { participation de celle-ci aux prises de décisions }\end{array}$ \\
\hline $\begin{array}{l}\text { Moyens de subsistance (développement) - vise le } \\
\text { changement en conjuguant les objectifs de l'utilisation } \\
\text { durable des terres et ceux du développement. }\end{array}$ & $\begin{array}{l}\text { Créer des débouchés économiques } \\
\text { grâce à la protection et/ou la } \\
\text { régénération des forêts et distribuer } \\
\text { les fruits du développement entre les } \\
\text { parties prenantes locales. }\end{array}$ & $\begin{array}{l}\text { Les revenus ou les bénéfices issus de la nouvelle } \\
\text { utilisation des terres compensent les pertes de } \\
\text { revenus subies par les parties prenantes locales } \\
\text { à la suite de l'abandon de leurs anciennes } \\
\text { pratiques. Ceci doit les motiver à mettre en } \\
\text { œuvre l'initiative. }\end{array}$ \\
\hline
\end{tabular}

Participation - vise le changement en accordant aux communautés plus de contrôle sur les ressources naturelles par l'intermédiaire des institutions locales, qui sont officielles et assimilées aux pouvoirs publics.
Attribuer aux communautés locales plus de contrôle sur leurs ressources grâce à la cogestion et au coapprentissage et/ou aux actions de renforcement des capacités.

Renforcer le capital social par la prise de décisions collaborative et la coordination à plusieurs niveaux.
Utilisation plus durable des terres, bénéfique pour les populations locales sur le plan économique et de nature à réduire leur vulnérabilité.

Processus participatif plus transparent et légitime, avec une population locale qui adhère davantage à l'initiative. par des initiatives impliquant différentes parties

prenantes et divers organismes gouvernementaux, issus de secteurs et d'échelons différents. pour les populations locales sur le plan économique et de nature à réduire leur vulnérabilité. Dans les 11 cas relevant de ce modèle, un dispositif a permis d'octroyer aux communautés locales plus de contrôle sur leurs ressources grâce à la cogestion et au coapprentissage et/ou aux actions de renforcement des capacités ${ }^{8}$.

\section{Coordination à plusieurs niveaux}

Ces forums cherchent à instaurer une utilisation plus durable des terres en mettant sur pied des initiatives rassemblant différentes parties prenantes qui opèrent à des échelons divers, notamment des organismes gouvernementaux. Dans les six cas de ce modèle, le dispositif mis en œuvre impliquait une coordination à plusieurs niveaux et une prise de décisions collaborative devant conduire à une utilisation plus durable des terres ${ }^{9}$. Cela devrait aussi donner lieu à un processus participatif plus transparent et légitime, impliquant davantage la population locale et donc l'adhésion à l'initiative.

\section{Vers des forums multipartites plus réactifs : enseignements dégagés}

Quatre facteurs interdépendants ont été identifiés comme caractéristiques des cas ayant au moins un peu réussi à encourager une participation véritable des acteurs les plus marginalisés et à remédier aux inégalités.meaningful participation of more marginalized actors and addressing inequality.

\footnotetext{
8 5/Népal CFP, 6/Bangkok Green, 7/Campo-Ma'an MF, 11/Finger Lakes, 12/ Népal DFCC, 13/Hin Nam No, 14/Nilhelmina MF, 15/Nusa Tenggara Barat, 16/ Île de Cardoso, 17/Prince Albert MF, 19/Manitoba MF.

9 10/Oddar Meanchey REDD+, 13/Hin Nam No, 14/Nilhelmina MF, 15/Nusa Tenggara Barat, 17/Prince Albert MF, 18/Papillon monarque.
}

Engagement envers la population, le processus et les objectifs, démontré par l'investissement en temps et en ressources et par un suivi complet permettant de veiller à l'application de la loi et des mesures décidées, et à la réalisation des objectifs de participation.

Parmi les cas, 13/Hin Nam No atteste de la volonté politique des pouvoirs publics de mettre en pratique la gestion collaborative d'une importante aire protégée, évolution suscitée par leur intérêt à attirer les touristes dans cette zone. Dans plusieurs cas (p. ex. 1/ Gadabanikilo JFM, 2/ Uttaranchal JFM, 3/Karnataka JFPM, 4/Karnataka JFM), le décalage entre la loi et la pratique était évident, car la population locale avait techniquement le droit de participer, mais les représentants de l'État et/ou les élites leur mettaient des bâtons dans les roues, ou l'investissement en temps et en ressources était insuffisant pour mettre en œuvre un forum multipartite. II est évident que, dans d'autres cas, la loi ne favorise pas du tout la participation.

Ces cas révèlent aussi l'importance du lien qui existe entre la pérennité d'un forum et la modulation de l'engagement (parfois imprévisible) en fonction de l'évolution au fil du temps des priorités de conservation et de développement des différentes parties prenantes. Cette revue de littérature montre que l'engagement et l'ouverture des pouvoirs publics sont nécessaires pour mettre en place des cadres juridiques permettant la décentralisation et l'inclusion de la population locale dans les processus décisionnels et pour veiller à leur mise en œuvre. Cela nous conduit au deuxième facteur.

Dialogue avec les pilotes de la mise en œuvre, notamment les principaux médiateurs et les représentants des échelons intermédiaires dans la hiérarchie des pouvoirs publics, qui déterminent ce qui se passe véritablement sur le terrain.

Dans certains cas (p. ex. 2/Uttaranchal JFM, 3/Karnataka JFPM), les initiatives ont échoué à cause de l'absence de rapport entre les buts apparents du projet et leurs modalités de réalisation par des animateurs moins impliqués que les concepteurs de ces buts dont 
ils faisaient une interprétation différente. À l'inverse, 6/Bangkok Green montre l'intérêt de mobiliser activement les responsables de l'aménagement au gouvernement local qui auraient pu sans cela être réticents à participer. Cela a été déterminant pour sa pérennité, grâce à l'instauration d'une confiance entre les communautés et le gouvernement, qui s'est traduite par un plan de maintien du projet à l'avenir. D'où l'importance d'harmoniser les engagements et les priorités tout en veillant à ce que ceux qui peuvent susciter le changement participent véritablement au forum multipartite (ou y soient effectivement représentés). Au fil du temps, cette approche peut sans doute mieux résister face aux changements de régimes politiques.

\section{S'informer auprès des parties prenantes et les écouter, surtout celles étant traditionnellement plus en retrait.}

II ressort clairement des cas que, si l'on organise les projets pour traiter les inégalités entre les sexes sans investir le temps et les ressources nécessaires, ni des méthodes fiables, les résultats attendus ne seront pas au rendez-vous. Le fait de mettre en avant la présence de femmes lors des réunions comme preuve de participation peut aggraver les problèmes, car cela pourrait légitimer un système de prise de décisions très inéquitable (1/Gadabanikilo JFM, 2/Uttaranchal JFM, 3/Karnataka JFPM). 7/Campo-Ma'an MF et 8/Dja et Mpomo MF montrent par comparaison les résultats productifs d'une volonté d'écouter, notamment les femmes dont la mobilisation pour réclamer un droit d'accès aux forêts a débouché sur l'évolution de certaines pratiques de gouvernance dans deux forêts modèles.

\section{Adaptabilité pour tenir compte des enseignements dégagés.}

L'adaptabilité va de pair avec l'apprentissage. L'apprentissage ne portera pas ses fruits si le processus n'est pas conçu pour s'adapter aux besoins de ceux qui sont en retrait par rapport à la prise de décisions, ou même pour changer les priorités du projet en vue de remettre en cause les institutions qui renforcent les inégalités. L'ouverture sur l'apprentissage et l'adaptation est capitale compte tenu de l'incidence éventuelle (positive ou non) des forums multipartites sur les institutions locales en place. Dans certains cas (p. ex. 3/Karnataka JFPM, 17/Prince Albert MF, 19/Manitoba MF), des populations vulnérables que les forums cherchaient à aider ont été perturbées par le fait de négliger les systèmes non officiels/informels de gestion des forêts et des ressources, comme la nature changeante des relations et leur histoire (notamment les déséquilibres de pouvoir). Le remplacement de ces modes de fonctionnement informel par des réglementations formelles et appliquées plus strictement peut accroître la vulnérabilité, même en cas de « participation » obligatoire. Pour être attentif aux institutions, y compris aux instances de représentation (Ribot 2007), il est nécessaire de les connaître et cela ne peut être à la portée des organisateurs et/ou des porteurs des projets de forums multipartites qu'après une période d'étude, de réflexion et de coapprentissage.

Ces quatre facteurs se conjuguent. Dans les cas où l'on a été attentif à l'apprentissage (p. ex. 5/Népal CFP, 6/Bangkok Green, 15/Nusa Tenggara Barat), cela a été possible grâce aux financements et au temps alloués. Ces forums multipartites ont donc pu être conçus sur un modèle intentionnellement adaptatif, qui privilégiait l'apprentissage (y compris la recherche scientifique), la prise en compte des disparités de pouvoir entre les parties prenantes (notamment sur le plan des connaissances) et qui préparait la population locale à participer. La confiance a pu s'installer entre les organisateurs des forums et les participants, et la volonté politique a pu se manifester pour mettre sur pied l'initiative, ce qui a permis aux parties prenantes de comprendre les conséquences du changement et les opportunités suscitées.

\section{La participation au centre du montage : lignes directrices pour changer l'approche des forums multipartites}

Le type de modèle proposé ici n'est ni strictement descendant ni ascendant. Pour mettre la participation au centre du montage, il faut un processus adaptatif avec des boucles de rétroaction entre le haut et le bas et vice versa. Cela nécessite un temps pour la recherche scientifique et les réunions à divers niveaux pour comprendre les difficultés potentielles que peuvent rencontrer les pilotes des projets locaux dans le contexte général dans lequel ils vont travailler. II s'avère utile de prendre le temps de rechercher et de recenser les parties prenantes et les institutions locales, les relations de pouvoir entre ces parties prenantes, et les voies du savoir, ces informations étant fondamentales pour concevoir des projets susceptibles de remédier aux inégalités existantes. Ce processus permet au consensus et à l'engagement de se forger à différents niveaux, qui vont se traduire par une volonté politique plus forte. Cela contribue aussi au renforcement des capacités et à l'autonomie des populations locales qui prendront davantage part aux processus décisionnels et défendront leurs droits.

Cette approche prévoit aussi l'apprentissage social qui est essentiel dans une gestion adaptative et comporte une grande part de réflexion (Berkes 2009). Lorsque le bilan du processus conduit les acteurs à réfléchir à leurs principes de départ et à les revoir, c'est ce qu'on appelle en anglais « double-loop learning », c'est-à-dire l'apprentissage en double boucle (Maarleveld et Dabgbégnon 1999). L'apprentissage « en triple boucle » (Romm et Flood 1996) conduit à changer de point de référence, où l'observateur quitte son expérience pour vivre celle de quelqu'un d'autre (Peschl 2007 ; Evans et al. sous presse). Ce type d'apprentissage demande de l'humilité de la part des organisateurs et des pilotes pour voir les populations locales comme partenaires dans la recherche de solutions plutôt que comme bénéficiaires des projets.

Pour autant, nous ne sommes pas en train d'affirmer qu'une intervention tenant compte de ces quatre facteurs est une solution miracle qui aplanira les inégalités. Les déséquilibres de pouvoir peuvent être tels que la population locale n'est pas en mesure de défendre sa position. D'après les cas étudiés, l'engagement, la participation, l'écoute et la volonté de changer sont les caractéristiques du processus qui devrait faire partie des forums multipartites ayant vocation à promouvoir une transformation au moyen de l'un des quatre modèles destinés à encourager l'utilisation durable des terres par la participation. Cela ne suffira sans doute pas à bousculer les institutions qui maintiennent la discrimination et les inégalités ( $p$. ex. rééquilibrer la répartition des ressources matérielles, Larson et Ribot 2007), mais ce serait déjà un 
grand pas dans la bonne direction si l'on comprenait le terrain de jeu pour mettre en place un mode de fonctionnement plus équitable. Ces forums ne résoudront pas tout, mais devraient plutôt s'inscrire dans une stratégie plus vaste.

À nos yeux, les sources de difficultés éventuelles sont les pratiques que nous observons fréquemment sur le terrain, ainsi que certaines tendances actuelles qui pourraient inspirer un mouvement contraire. L'une d'elles est la nature de plus en plus court-termiste des financements octroyés par les bailleurs de fonds et, dans le même temps, le rattachement des financements à des indicateurs d'impact simples et quantifiables. La première tendance va à contre-courant des constatations de cette étude, tandis que la seconde, en se focalisant uniquement sur les chiffres, ne permet pas de constater les changements positifs. Un autre souci est l'urgence impérieuse de la lutte contre le changement climatique, car les appels à agir immédiatement peuvent renforcer les interventions descendantes au détriment de la participation, de l'écoute et de l'apprentissage (Hulme 2011).

Il conviendrait de faire pression sur les bailleurs de fonds pour qu'ils privilégient les investissements à plus long terme et les financements souples susceptibles d'encourager la généralisation de ces démarches de participation et de s'adapter à de nouvelles priorités négociées. Les praticiens devraient avoir l'humilité implicite en apprentissage adaptatif et être ouverts à l'apprentissage en triple boucle. Dans les projets, il faudrait prévoir de travailler avec la population locale pour recenser et analyser les relations institutionnelles et de pouvoir, ainsi que d'autres facteurs contextuels. Une analyse poussée permettra de jeter les bases nécessaires à la remise en cause des relations de pouvoir entravant souvent les processus multipartites.

\section{Remerciements}

Les auteurs remercient Christopher Hewlett et Deborah Delgado, leurs coauteurs pour leur synthèse réaliste (Realist Synthesis Review). Ils sont aussi reconnaissants aux relecteurs anonymes de World Development ainsi qu'à Jesse C. Ribot, Jonathan Fox et Melanie McDermott pour leurs commentaires sur cet Infobrief. Cette étude a été financée par l'Agence norvégienne de coopération pour le développement, la Commission européenne, I'Initiative internationale pour le climat du ministère fédéral allemand de l'Environnement, de la Conservation de la Nature et de la Sûreté nucléaire et le ministère du Développement international du Royaume-Uni. Ces travaux ont été entrepris dans le cadre de deux programmes de recherche du CGIAR: celui sur les politiques, les institutions et les marchés (PIM), conduit par I'Institut International de Recherche sur les Politiques Alimentaires (IFPRI), et celui sur les forêts, les arbres et l'agroforesterie (FTA) qui est dirigé par le CIFOR. Les opinions exprimées ici n'engagent que les auteurs et ne correspondent pas forcément à la position de l'IFPRI, du CIFOR, du CGIAR ou des bailleurs de fonds.

\section{Bibliographie}

Berkes F. 2009 Evolution of co-management: Role of knowledge generation, bridging organizations and social learning. Journal of Environmental Management 90:1692-1702.
Boyd W, Stickler C, Duchelle AE, Seymour F, Nepstad D, Bahar NHA et Rodriguez-Ward D. 2018 Jurisdictional approaches to REDD+ and low emissions development: Progress and prospects. Document de travail. Washington, DC: World Resources Institute.

Chambers R. 1983 Rural Development: Putting the Last First. Harlow, États-Unis : Prentice Hall.

Cornwall A. 2004 New Democratic Spaces? The Politics and Dynamics of Institutionalised Participation. IDS Bulletin 35(2):1-10. Brighton, Royaume-Uni : Institute of Development Studies.

Espinoza Llanos R et Feather C. 2011 The reality of REDD+ in Peru: Between theory and practice. Lima: AIDESEP et Forest Peoples Programme.

Evans K, Larson AM et Flores S. Sous presse. Learning to learn in tropical forests: training field teams in adaptive collaborative management, monitoring and gender. International Forestry Review.

Faysse N. 2006 Troubles on the way: An analysis of the challenges faced by multi-stakeholder platforms. Natural Resource Forum. 30. 219-229.

Flood R et Romm N.1996 Contours of diversity management and triple loop learning. Kybernetes 25:154-63.

Hulme M. 2011 Reducing the Future to Climate: A Story of Climate Determinism and Reductionism. Osiris 26:245-66.

Kusters K, Buck L, de Graaf M, Minang P, van Oosten C et Zagt R. 2018 Participatory Planning, Monitoring and Evaluation of Multi-Stakeholder Platforms in Integrated Landscape Initiatives. Environmental Management 62(1):170:81.

Larson AM et Ribot JC. 2007 The poverty of forestry policy: Double standards on an uneven playing field. Sustainability Science 2(2):189-204.

Nilsson D, Baxter G, Butler JRA et McAlpine CA. 2016 How do community-based conservation programs in developing countries change human behaviour? A realist synthesis. Biological Conservation 200:93-103.

Maarleveld M et Dabgbégnon C. 1999 Managing natural resources: A social learning perspective. Agriculture and Human Values 16:267-80.

McLain R, Lawry S et Ojanen M. 2018 Fisheries' property regimes and environmental outcomes: A realist synthesis review. World Development 102:213-27.

Mena S et Palazzo G. 2012 Input and output legitimacy of multistakeholder initiatives. Business Ethics Quarterly 22(3):527-56.

Peschl MF. 2007 Triple-loop learning as foundation for profound change, individual cultivation, and radical innovation: Construction processes beyond scientific and rational knowledge. Constructivist Foundations 2:136-45.

Ravikumar A, Larson AM, Myers R et Trench T. 2018 Inter-sectoral and multilevel coordination alone do not reduce deforestation and advance environmental justice. Environment and Planning C: Politics and Space 36(8):1437-57.

Ribot JC. 2007 Representation, citizenship and the public domain in democratic decentralization. Development 50:43-49.

Sarmiento Barletti JP, Larson AM, Hewlett C et Delgado D. 2020 Designing for engagement: A realist synthesis review of how context affects the outcomes of multi-stakeholder forums on land use and/or land-use change. World Development 127. doi. org/10.1016/j.worlddev.2019.104753

Sarmiento Barletti JP et Larson AM. 2020 Models of participation in multi-stakeholder forums: Results of a Realist Synthesis Review. CIFOR Infobrief 281. Bogor, Indonésie : CIFOR.

Sarmiento Barletti JP, Hewlett C et Larson AM. 2018 Protocol for a realist synthesis review: How does context affect the outcomes of subnational 
multi-stakeholder forums on land use and/or land-use change? Bogor, Indonésie : CIFOR.

Stickler C, Duchelle AE, Ardila JP, Nepstad D, David O, Chan C, Rojas JG, Vargas R, Bezerra T, Pritchard L, et al. 2018 The state of jurisdictional sustainability: Synthesis for practitioners and policymakers. San Francisco, États-Unis : Earth Innovation Institute.

Young I. 2000 Inclusion and Democracy. New York: Oxford University Press.

\section{Études de cas}

Brenner L et Job H. 2012 Challenges to actor-oriented environmental governance: Examples from three Mexican biosphere reserves. Tijdschrift voor Economische en Sociale Geografie 103(1):1-19.

Butler JR, Suadnya W, Yanuartati Y, Meharg S, Wise RM, Sutaryono Y et Duggan K. 2016 Priming adaptation pathways through adaptive co-management: Design and evaluation for developing countries. Climate Risk Management 12:1-16.

de Koning M, Nguyen T, Lockwood M, Sengchanthavong S et Phommasane S. 2017 Collaborative governance of protected areas: Success factors and prospects for Hin Nam No Natural Protected Area, central Laos. Conservation \& Society 15(1):87-99.

Gebara MF. 2013 Importance of local participation in achieving equity in benefit-sharing mechanisms for REDD+: A case study from the Juma Sustainable Development Reserve. International Journal of the Commons 7(2):473-97.

Jum CN, Nguiebouri J, Zoa M et Diaw C. 2007 Building broad-based partnership for sustainable forest management: The Model Forest experience in Cameroon. International Journal of Environmental Studies 64(5):625-41.

Klenk NL, Reed MG, Lidestav G et Carlsson J. 2013 Models of representation and participation in Model Forests: Dilemmas and implications for networked forms of environmental governance involving indigenous people. Environmental Policy and Governance 23(3):161-76.

Martin A et Lemon M. 2001 Challenges for participatory institutions: The case of village forest committees in Karnataka, South India. Society \& Natural Resources 14(7):585-97.

McDougall C, Jiggins J, Pandit BH, Thapa Magar Rana SK et Leeuwis C. 2013 Does adaptive collaborative forest governance affect poverty? Participatory action research in Nepal's community forests. Society \& Natural Resources 26(11):1235-51.

Mohanty R. 2004 Institutional dynamics and participatory spaces: The making and unmaking of participation in local forest management in India. IDS Bulletin 35(2):26-32.

Nayak PK et Berkes F. 2008 Politics of co-optation: Community forest management versus joint forest management in Orissa, India. Environmental Management 41(5):707-18

Parkins JR, Dunn M, Reed MG et Sinclair AJ. 2016 Forest governance as neoliberal strategy: A comparative case study of the Model Forest Program in Canada. Journal of Rural Studies 45:270-78.

Pasgaard M. 2015 Lost in translation? How project actors shape REDD+ policy and outcomes in Cambodia. Asia Pacific Viewpoint 56(1):111-27.

Rana B, Khanal KP, Kotru R et Jamarkattel B. 2009 Tackling the Terai forest governance impasse: Can district-level multi-stakeholder processes help? Journal of Forest and Livelihood 8(2):16-26.

Sessin-Dilascio K, Prager K, Irvine KN et de Almeida Sinisgalli PA. 2015 The dynamics of co-management and social capital in protected area management - The Cardoso Island State Park in Brazil. World Development 67:475-89.

Stringer LC, Dougill AJ, Fraser E, Hubacek K, Prell C et Reed MS. 2006 Unpacking 'participation' in the adaptive management of socialecological systems: A critical review. Ecology and Society 11(2):39.

Twarkins M, Fisher L et Robertson T. 2001 Public involvement in forest management planning: A view from the Northeast. Journal of Sustainable Forestry 13(1/2):237-51.

\begin{tabular}{|c|c|c|}
\hline CGIAR & $\begin{array}{l}\text { RESEARCH } \\
\text { PROGRAM ON } \\
\text { Policies, } \\
\text { Institutions, } \\
\text { and Markets } \\
\quad \text { Led by IFPRI }\end{array}$ & $\begin{array}{l}\text { Le programme de recherche du CGIAR sur les politiques, les institutions et les marchés (PIM) a pour objet la } \\
\text { recherche-action pour équiper les instances de décision des informations nécessaires à l'élaboration de } \\
\text { politiques agricoles et alimentaires qui servent les intérêts des producteurs pauvres et des consommateurs, } \\
\text { hommes et femmes. Le PIM s'appuie sur les ressources des centres du CGIAR et de nombreux partenaires } \\
\text { nationaux, régionaux et internationaux. Ce programme est dirigé par l'Institut International de Recherche sur } \\
\text { les Politiques Alimentaires (IFPRI). www.pim.caiar.org }\end{array}$ \\
\hline
\end{tabular}

\begin{tabular}{|l|l}
\hline PROGRAMME DE \\
CGECERCHE SUR \\
les Forêts, les Arbres et
\end{tabular}

Cette recherche a été menée par le CIFOR dans le cadre du Programme de Recherche du CGIAR sur les Forêts, les Arbres et l'Agroforesterie (FTA). Le FTA constitue le plus important programme global de recherche pour le développement visant à amplifier la contribution des forêts, des arbres et de l'agroforesterie au développement durable, à la sécurité alimentaire et à la lutte contre le changement climatique. Le CIFOR dirige le FTA en partenariat avec Bioversity International, le CATIE, le CIRAD, I'ICRAF, I'INBAR et TBI. Les travaux du programme FTA sont soutenus par le Fonds fiduciaire du CGIAR : cgiar.org/funders

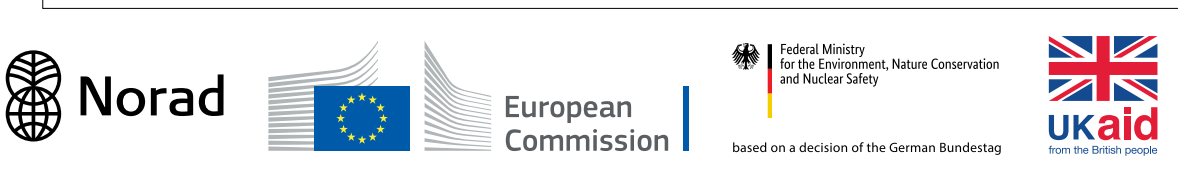

